\title{
Transcriptome Analysis of Porcine PBMCs Reveals the Immune Cascade Response and Gene Ontology Terms Related to Cell Death and Fibrosis in the Progression of Liver Failure
}

\author{
YiMin Zhang, ${ }^{1}$ Li Shao, ${ }^{1}$ Ning Zhou, ${ }^{1}$ JianZhou Li, ${ }^{1}$ Yu Chen, ${ }^{2}$ Juan Lu, ${ }^{1}$ Jie Wang, \\ ErMei Chen, ${ }^{1}$ ZhongYang Xie, ${ }^{1}$ and LanJuan Li ${ }^{1}{ }^{1}$ \\ ${ }^{1}$ State Key Laboratory for Diagnosis and Treatment of Infectious Diseases, \\ Collaborative Innovation Center for Diagnosis and Treatment of Infectious Diseases, The First Affiliated Hospital, \\ College of Medicine, Zhejiang University, Hangzhou, Zhejiang Province, China \\ ${ }^{2}$ Department of Experimental Animals, Zhejiang Academy of Traditional Chinese Medicine, Hangzhou, Zhejiang Province, China
}

Correspondence should be addressed to LanJuan Li; ljli@zju.edu.cn

Received 22 January 2018; Accepted 4 March 2018; Published 12 April 2018

Academic Editor: En-Qiang Chen

Copyright (c) 2018 YiMin Zhang et al. This is an open access article distributed under the Creative Commons Attribution License, which permits unrestricted use, distribution, and reproduction in any medium, provided the original work is properly cited.

Background. The key gene sets involved in the progression of acute liver failure (ALF), which has a high mortality rate, remain unclear. This study aims to gain a deeper understanding of the transcriptional response of peripheral blood mononuclear cells (PBMCs) following ALF. Methods. ALF was induced by D-galactosamine (D-gal) in a porcine model. PBMCs were separated at time zero (baseline group), $36 \mathrm{~h}$ (failure group), and $60 \mathrm{~h}$ (dying group) after D-gal injection. Transcriptional profiling was performed using RNA sequencing and analysed using DAVID bioinformatics resources. Results. Compared with the baseline group, 816 and 1,845 differentially expressed genes (DEGs) were identified in the failure and dying groups, respectively. A total of five and two gene ontology (GO) term clusters were enriched in 107 GO terms in the failure group and 154 GO terms in the dying group. These GO clusters were primarily immune-related, including genes regulating the inflammasome complex and toll-like receptor signalling pathways. Specifically, GO terms related to cell death, including apoptosis, pyroptosis, and autophagy, and those related to fibrosis, coagulation dysfunction, and hepatic encephalopathy were enriched. Seven Kyoto Encyclopedia of Genes and Genomes (KEGG) pathways, cytokine-cytokine receptor interaction, hematopoietic cell lineage, lysosome, rheumatoid arthritis, malaria, and phagosome and pertussis pathways were mapped for DEGs in the failure group. All of these seven KEGG pathways were involved in the 19 KEGG pathways mapped in the dying group. Conclusion. We found that the dramatic PBMC transcriptome changes triggered by ALF progression was predominantly related to immune responses. The enriched GO terms related to cell death, fibrosis, and so on, as indicated by PBMC transcriptome analysis, seem to be useful in elucidating potential key gene sets in the progression of ALF. A better understanding of these gene sets might be of preventive or therapeutic interest.

\section{Introduction}

Acute liver failure (ALF) is a severe syndrome characterised by hepatic encephalopathy and coagulation dysfunction, which can lead to multiorgan failure and death [1-3]. High morbidity and mortality following ALF are major problems worldwide $[2,3]$. Thus, a thorough understanding of key genes or gene sets that regulate the progression of ALF is required.
The development of second-generation sequencing, particularly RNA-sequencing (RNA-Seq), has made it possible to perform global analysis of changes in gene expression during the course of a disease [4-6].

Taking biopsy samples during an ALF flare places the patient at high risk for lethal bleeding. More importantly, biopsy would influence the progression of ALF.

Analysis of the transcriptome of peripheral blood mononuclear cells (PBMCs) has successfully elucidated the 
mechanisms of numerous complex diseases and vaccination models [7-10]. These studies showed that analysing the PBMC transcriptome is helpful in identifying key genes and gene sets that control disease progression.

Here, we performed a comparative analysis of PBMC transcriptome in a porcine model of D-galactosamine- (Dgal-) induced ALF to identify candidate genes and gene sets that play important roles in the progression of ALF.

\section{Materials and Methods}

2.1. Porcine Model of D-gal-Induced ALF. A D-gal-induced ALF porcine model was used as previously described by our group [11]. Briefly, male Bama experimental miniature pigs were used and $1.3 \mathrm{~g} / \mathrm{kg}$ body weight D-gal (Hanhong Chemical, Shanghai, China) was intravenously injected to induce ALF. Blood samples were collected at baseline (time zero) and 36 and $60 \mathrm{~h}$ after D-gal injection. Pigs were sacrificed after blood sample collection at $60 \mathrm{~h}$. The general medical condition of the experimental pigs was monitored throughout the experiment.

All animal experiments were conducted in the Department of Experimental Animals, Zhejiang Academy of Traditional Chinese Medicine, China, and approved by the Animal Care Ethics Committee of the Academy. All experimental animals were treated humanely.

2.2. Clinical Parameters following D-gal-Induced Porcine ALF. At 0,36 , and $60 \mathrm{~h}$, parameters to quantify the severity of liver failure were collected including the international normalization ratio (INR), and alanine aminotransferase, aspartate aminotransferase, alkaline phosphatase, $\gamma$-glutamyl transpeptidase, total bilirubin, and creatinine levels.

Blood ammonia was measured using an ammonia test kit (ARKRAY, Tokyo, Japan) with a detection range between 10 and $400 \mu \mathrm{g} / \mathrm{dL}$. INR was quantified using STA-R (Diagnostic Stago, Asnieres, France) in the emergency laboratory at the First Affiliated Hospital, College of Medicine, Zhejiang University. Serum alanine aminotransferase, aspartate aminotransferase, alkaline phosphatase, $\gamma$-glutamyl transpeptidase, total bilirubin, and creatinine levels were measured using an automated biochemical analyser (Abbott Aeroset; Abbott Laboratories, Chicago, IL, USA) in the same laboratory.

2.3. PBMC Isolation and RNA Extraction. PBMCs were isolated using Ficoll-Histopaque (Sigma Aldrich, St. Louis, MO, USA) immediately after blood sample collection. Subsequently, total RNA was extracted using RNeasy Mini kits (QIAGEN, Hilden, Germany) according to the manufacturer's instructions. All RNA samples were stored at $-80^{\circ} \mathrm{C}$ for future analysis.

2.4. mRNA Library Construction, RNA-Sequencing, and Data Analysis. Total RNA ( $1 \mu \mathrm{g})$ was thawed to create a library using TruSeq Stranded RNA LT Guide (Illumina, San Diego, CA, USA) according to the manufacturer's instructions. An Agilent 2100 bioanalyser (Santa Clara, CA, USA) was used to evaluate the concentration and size distribution of complementary DNA (cDNA) in the library before sequencing with the Illumina HiSequation 2500 system. The high-throughput sequencing was performed according to the manufacturer's instructions (Illumina HiSequation 2500 User Guide).

The raw data were filtered by FASTX (ver. 0.0.13) before mapping to the genome using TopHat (ver. 2.0.9). Gene fragments were counted using HTSeq followed by trimmed mean of $M$ values (TMM) normalization. Significantly differentially expressed genes (DEGs) were identified using Cufflinks (ver. 2.2.1) [12]. DEGs were then submitted to Visualisation and Integrated Discovery analysis (DAVID; ver. 6.8) [13] for gene ontology (GO) term enrichment and clustering and Kyoto Encyclopedia of Genes and Genomes (KEGG) pathway mapping using default parameters, except for an EASE score setting of 0.05 .

2.5. Validation of RNA-Seq Data by $q R T-P C R$. Quantitative RT-PCR was performed on selected genes to validate the data obtained from mRNA sequencing. Briefly, total RNA was reverse-transcribed into cDNA using the Fast Quant RT kit (Tiangen, Beijing, China). All qRT-PCR was conducted using SYBR Green SuperReal PreMix Plus (FP205; Tiangen) on an ABI 7900HT (Applied Biosystems, Foster City, CA, USA). Experimental conditions included a 3-min cycle at $94^{\circ} \mathrm{C}$ followed by 40 cycles of $20 \mathrm{~s}$ at $94^{\circ} \mathrm{C}, 20 \mathrm{~s}$ at $58^{\circ} \mathrm{C}$, and $20 \mathrm{~s}$ at $72^{\circ} \mathrm{C}$.

Each qRT-PCR run was performed in triplicate with two biological replicates. Beta-2-microglobulin (B2M) was used as the reference gene for data normalization, as previously described. A correlation analysis of the fold change of selected genes between qRT-PCR and RNA-Seq was performed.

2.6. Statistical Analysis. RNA-seq data analyses were described previously in Section 2.4. Other statistical analyses were performed by Graphpad Prism (Version 5.0, GraphPad Software, San Diego, United States). Biochemical parameters in the progress of ALF were compared using Student's $t$-test. Linear regression was performed in validation of RNA-Seq data by qRT-PCR. A $p$ value less than 0.05 was considered significant.

\section{Results}

3.1. Clinical Features and Biochemical Parameters of D-galInduced ALF in Pigs. All animals enrolled in this experiment were healthy, with a good appetite and response to the Dgal injection at time zero (baseline). The ALF model was successfully established in all the animals at $36 \mathrm{~h}$ (failure) after D-gal injection. The pigs stopped eating and became obviously restless, with yellow urine. At $60 \mathrm{~h}$ post-injection (dying), the pigs showed ataxia and symptoms of hepatic encephalopathy, with no reaction to painful stimuli.

The biochemical parameters as ALF progressed are listed in Table 1. Liver failure was identified by the progressive increase in liver enzymes, bilirubin, blood ammonia, and the international normalization ratio in both the failure and dying groups as compared to the baseline group. A deviation of bilirubin and liver enzymes, or elevated total bilirubin with decreased liver enzymes, was observed in the dying group but not in the failure group. 
TABLE 1: Biochemical parameters in a porcine model of ALF.

\begin{tabular}{lccc}
\hline Parameters & Baseline & Failure & Dying \\
\hline International normalization ratio & $0.9 \pm 0.05$ & $2.7 \pm 0.2^{* *}$ & $4.8 \pm 0.8^{* *}$ \\
Ammonia $(\mu \mathrm{g} / \mathrm{dl})$ & $22.3 \pm 3.1$ & $76.5 \pm 8.7^{* *}$ & $225.5 \pm 47.4^{* *}$ \\
Alanine aminotransferase $(\mathrm{U} / \mathrm{L})$ & $56.3 \pm 8.0$ & $311.5 \pm 65.0^{*}$ & $230.3 \pm 46.5^{*}$ \\
Aspartate aminotransferase $(\mathrm{U} / \mathrm{L})$ & $36.0 \pm 3.3$ & $5023.8 \pm 1034.6^{*}$ & $1788.5 \pm 263.6^{* *}$ \\
Alkaline phosphatase $(\mathrm{U} / \mathrm{L})$ & $72.8 \pm 16.9$ & $232.8 \pm 53.4^{*}$ & $564.0 \pm 82.6^{* *}$ \\
$\gamma$-Glutamyl transpeptidase $(\mathrm{U} / \mathrm{L})$ & $64.5 \pm 9.6$ & $77.0 \pm 5.1$ & $96.3 \pm 5.0^{*}$ \\
Total bilirubin $(\mu \mathrm{mol} / \mathrm{L})$ & $2.3 \pm 0.3$ & $40.8 \pm 5.7^{* *}$ & $70.8 \pm 7.6^{* *}$ \\
Creatinine $(\mathrm{mmol} / \mathrm{L})$ & $58.0 \pm 2.1$ & $59.3 \pm 6.4$ & $49.5 \pm 3.3$ \\
\hline
\end{tabular}

Data are means \pm SEM. ${ }^{*} p<0.05,{ }^{* *} p<0.01$ versus baseline.

TABLE 2: Qualitative analysis of PBMC RNA-Seq data in a porcine model of ALF.

\begin{tabular}{|c|c|c|c|c|c|c|c|}
\hline Sample name & Raw reads & Q20 value & Clean reads & Mapped reads & Genic reads & Percentage of genic reads & Expressed gene number \\
\hline Baseline-1 & $80,876,352$ & $94.80 \%$ & $77,508,054$ & $63,736,589$ & $51,530,237$ & $80.80 \%$ & 15,249 \\
\hline Baseline-2 & $122,478,648$ & $95.40 \%$ & $117,994,584$ & $64,288,782$ & $47,627,719$ & $74.10 \%$ & 14,990 \\
\hline Baseline-3 & $97,633,918$ & $95.10 \%$ & $93,252,338$ & $76,290,007$ & $48,400,655$ & $63.40 \%$ & 15,470 \\
\hline Baseline-4 & $91,203,498$ & $95.30 \%$ & $87,519,976$ & $70,759,871$ & $54,612,207$ & $77.20 \%$ & 15,642 \\
\hline Dying-1 & $106,182,528$ & $95.20 \%$ & $101,728,266$ & $83,553,821$ & $57,816,273$ & $69.20 \%$ & 15,563 \\
\hline Dying-2 & $93,997,436$ & $95.20 \%$ & $90,503,598$ & $74,471,193$ & $60,365,900$ & $81.10 \%$ & 15,101 \\
\hline Dying-3 & $118,901,914$ & $94.90 \%$ & $91,408,790$ & $73,952,809$ & $57,686,330$ & $78.00 \%$ & 15,712 \\
\hline Dying- 4 & $84,848,674$ & $95.30 \%$ & $81,623,206$ & $67,004,468$ & $55,869,637$ & $83.40 \%$ & 15,343 \\
\hline Failure-1 & $80,262,120$ & $95.20 \%$ & $76,885,638$ & $63,014,127$ & $49,808,658$ & $79.00 \%$ & 15,297 \\
\hline Failure-2 & $100,438,952$ & $95.30 \%$ & $100,291,397$ & $82,963,827$ & $68,021,072$ & $82.00 \%$ & 15,812 \\
\hline Failure-3 & $86,394,661$ & $94.90 \%$ & $66,295,638$ & $54,080,154$ & $38,129,055$ & $70.50 \%$ & 15,228 \\
\hline Failure-4 & $99,697,480$ & $94.80 \%$ & $95,927,678$ & $79,312,204$ & $60,637,999$ & $76.50 \%$ & 15,404 \\
\hline
\end{tabular}

3.2. Statistical Analysis of PBMC Transcriptome Data. RNASeq was performed in a total of 12 samples, with 4 samples in each group (baseline, failure, and dying). More than $9 \mathrm{~Gb}$ sequence data was the yield in each sample. Overall, 80.3-122.5 million raw reads per sample were generated with the quality of over $94.8 \%$ Q20, in which $66.3-118.0$ million were clean reads.

A total of 54.1-83.5 million reads were mapped to the porcine genome, in which $63.4-83.4 \%$ fell in genic regions while the remaining were in intergenic regions. 14,990 to 15,812 expressed genes were identified (fragments per kilobase of exon per million mapped reads [FPKM] >0) in each sample, respectively. Detailed information is presented in Table 2.

3.3. Differential Expression of Genes Associated with the Progression of D-gal-Induced ALF. DEGs during progression of D-gal-induced ALF were identified using Cufflinks (ver. 2.2.1). Genes were identified as significantly different with a false discovery rate (FDR) when the adjusted $p$ value was $(<0.05)$ and a greater than twofold log change was evident. Compared to the baseline group, 816 DEGs (Supplementary Table 1) were identified in the failure group and 1,845 DEGs (Supplementary Table 2) were identified in the dying group. A total of 590 identified genes overlapped between the two groups. Details are presented in Figure 1.

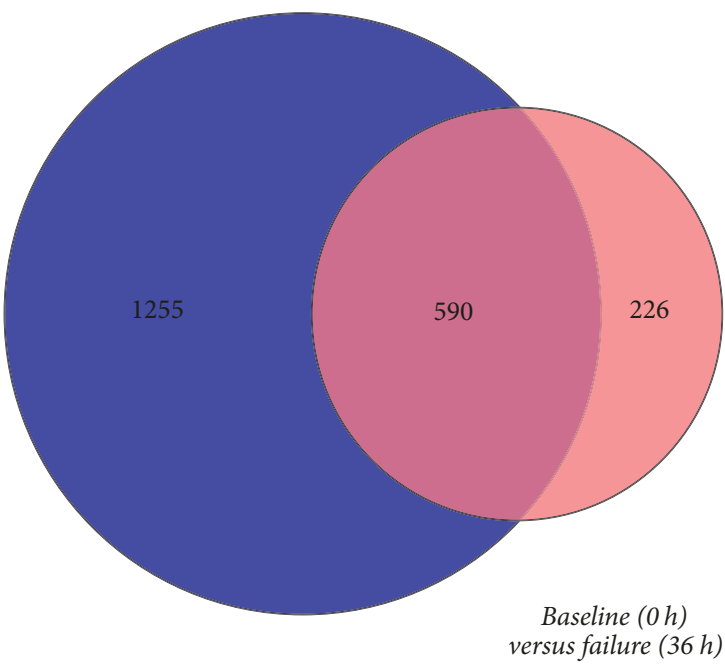

Baseline $(0 \mathrm{~h})$ versus dying $(60 \mathrm{~h})$

FIGURE 1: Differential expression of genes involved in the progression of acute liver failure (ALF).

3.4. Progression of D-gal-Induced ALF: GO Analysis. GO enrichment analysis and term clustering were performed to identify DEGs in the failure and dying groups as compared 
to the baseline group. In total, 107 GO terms were enriched for DEGs identified in the failure group, of which 76 were within the biological process (BP) category, 15 were within the cellular component (CC) category, and 16 were within the molecular function (MF) category. Among these GO terms, 26 were grouped into five independent clusters. The GO terms in the five clusters were related to positive regulation of the inflammatory response, the inflammasome complex, the toll-like receptor (TLR) signalling pathway, cell chemotaxis, and semaphorin receptor activity. With the exception of predominantly innate immune-related terms, important GO terms related to cell death were also enriched for processes such as apoptosis and pyroptosis. GO terms related to autophagy, another type of programmed cell death, were also identified. These terms are the regulation of autophagy, phagocytic vesicles, and lysosomes. Terms related to the process of liver fibrosis included gene sets important in the negative regulation of the fibroblast growth factor receptor signalling pathway and semaphorin receptor activity, and so on. Moreover, GO terms related to coagulation dysfunction and hepatic encephalopathy were also enriched, such as blood coagulation, astrocyte development, and the semaphorinplexin signalling pathway involved in axon guidance, branchiomotor neuron axon guidance, and so on.

In total, $154 \mathrm{GO}$ terms were enriched for the DEGs identified in the dying group, which included 104 BP terms, 25 $\mathrm{CC}$ terms, and $25 \mathrm{MF}$ terms. Overall, 20 out of $154 \mathrm{GO}$ terms were included in two clusters. The representative GO terms in these clusters were related to regulation of the inflammatory response and the inflammasome complex. Most enriched GO terms were predominantly immune-related. Other important GO terms were related to cell death, including apoptotic processes, apoptotic-signalling pathways, negative regulation of apoptotic processes, pyroptosis, autophagy, lysosomal membrane, and lysosomal lumen. Fibrosis-related terms such as collagen catabolic processes and collagen binding were also enriched; hepatic encephalopathy-related GO term, astrocyte development, was also enriched.

Details of GO enrichment and clustering are presented in Figure 2 .

3.5. KEGG Pathways Involved in the Progression of D-galInduced $A L F$. KEGG pathway mapping was used to study the molecular interactions and relation networks of the identified DEGs participating in metabolism, cellular processes and so on following D-gal-induced ALF. A total of seven KEGG pathways were mapped from DEGs identified in the failure group, all of which overlapped with the 19 identified KEGG pathways in the dying group. The seven KEGG pathways that were common to both included cytokine-cytokine receptor interaction, hematopoietic cell lineage, lysosome, rheumatoid arthritis, malaria, phagosome, and pertussis pathways. The remaining 12 KEGG pathways identified in the dying group were predominantly immune-related pathways, such as the NF-kappa B signalling pathway, the tumour necrosis factor (TNF) signalling pathway, and the complement and coagulation cascade pathways. KEGG pathways of diseases characterised by impaired liver function, such as Chagas disease (American trypanosomiasis), Salmonella infection, and Legionellosis, were also mapped using KEGG pathway mapping. Details are presented in Table 3.

3.6. Validation of RNA-Seq Data by qRT-PCR Analysis. To validate the RNA-Seq data, qRT-PCR of 12 selected genes was performed. The forward and reverse pairs of qRT-PCR primers for each gene are listed in Supplementary Table 3. Linear correlation analysis was conducted between the RNASeq and qRT-PCR results, which showed that the fold changes were significantly concordant between RNA-Seq and qRTPCR data $(r=0.95, p<0.0001)$. Results are shown in Figure 3 and Supplementary Table 4.

\section{Discussion}

ALF is a syndrome characterised by severe coagulopathy due to liver dysfunction and altered consciousness as a result of hepatic encephalopathy [3]. These features of ALF can be revealed at the PBMC level by transcriptome analysis, with the enriched GO term of blood coagulation and the mapped KEGG pathway of complement and coagulation cascades. Vemuganti et al. reported that, in association with hepatic encephalopathy, axon guidance micro-RNA levels changed in the cerebral cortex of a rat model of ALF [14]. In this study, three GO terms-branchiomotor neuron axon guidance, the semaphorin-plexin signalling pathway involved in axon guidance, and the cortical cytoskeleton-were identified. KEGG mapping analysis also identified disease-related KEGG pathways characterised by liver dysfunction, such as malaria, Chagas disease (American trypanosomiasis), Salmonella infection, and Legionellosis. The ability of PBMCs to migrate in a transendothelial manner and establish a dialogue between cells in solid organs has been reported previously [15-17]. These findings may explain the transcriptome changes observed in PBMCs that parallel the changes observed in solid organs, such as the liver and brain.

Previous studies have revealed extensive differential gene expression detected in the liver during the progression of ALF [18]. In this study, compared to the baseline group, the number of DEGs identified in the dying group was more extensive than in the failure group (1845 and 816 genes, resp.), which suggests that the cascades identified by PBMC transcriptome analysis change as ALF progresses. In addition, seven common KEGG pathways were identified for DEGs in both the failure and dying groups, which showed that the key pathways triggered by ALF result in further cascades at the transcriptome level.

Systemic inflammatory responses play an important role in the progression of ALF. Several key innate and adaptive immune mechanisms of ALF have been described previously, including acquired neutrophil dysfunction [19, 20], TLR function [21-23], and the important actions of chemokine and cytokine storms [24, 25]. All of these immune-related changes were identified in our GO enrichment and KEGG pathway mapping studies, which included genes involved in neutrophil chemotaxis, the TLR signalling pathway, and the TNF signalling pathway, among others.

Cell death plays an important role in ALF [26, 27]. Apoptosis is a form of hepatocyte death that contributes to 


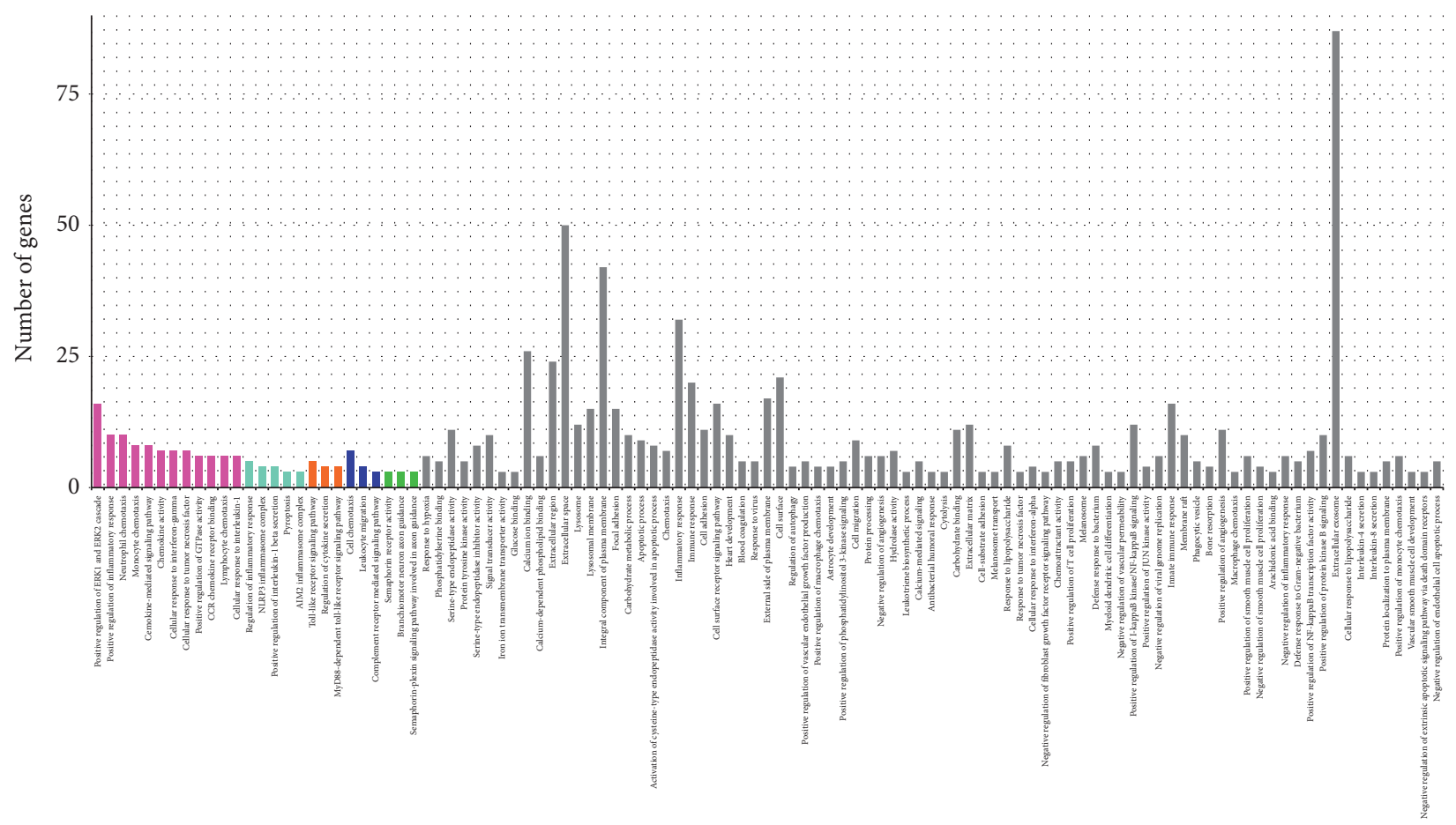

(a)

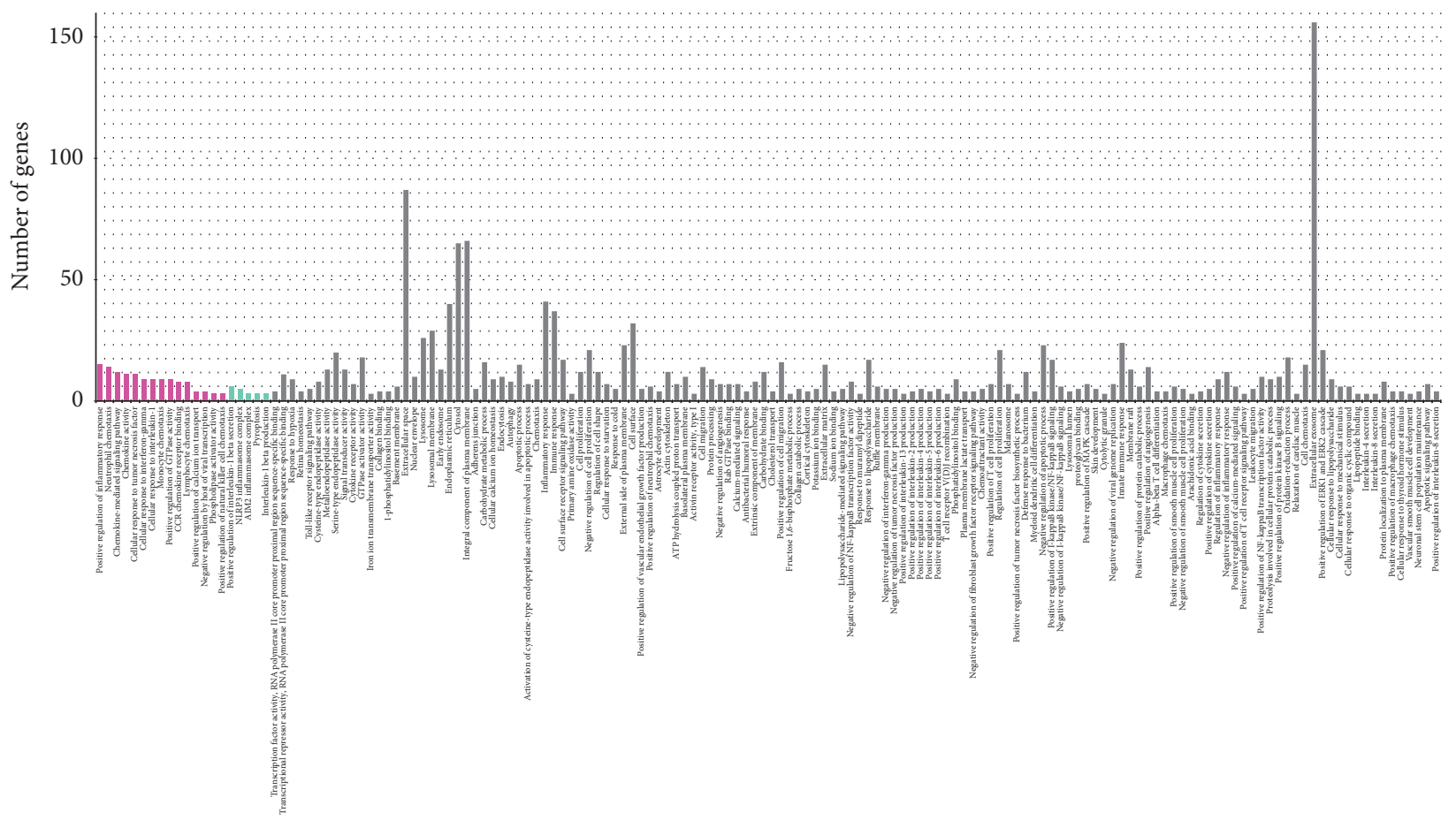

(b)

Figure 2: (a) Gene ontology (GO) terms of differentially expressed genes (DEGs) in the failure versus baseline group. (b) GO terms of DEGs in the dying versus baseline group.

ALF [28, 29]. Evidence of apoptotic pathways was identified in our transcriptome analysis. Two GO terms, the apoptotic process and apoptotic-signalling pathways, were enriched. Apart from apoptosis, recent research has focused on a new form of proinflammatory cell death known as pyroptosis [30].
Until now, studies on pyroptosis in ALF have been limited $[31,32]$. Furthermore, to the best of our knowledge, a role for pyroptosis in drug-induced ALF has not been reported previously. The enrichment of pyroptosis GO terms following D-gal-induced ALF suggests that pyroptosis is an important 
TABLE 3: KEGG pathways involved in the progression of ALF.

\begin{tabular}{|c|c|c|c|c|c|c|}
\hline \multirow[b]{2}{*}{ Name } & \multicolumn{3}{|c|}{ Failure versus baseline } & \multicolumn{3}{|c|}{ Dying versus baseline } \\
\hline & $\begin{array}{c}\text { Mapped } \\
\text { genes }\end{array}$ & $\begin{array}{c}\text { Fold } \\
\text { enrichment }\end{array}$ & $\begin{array}{c}\text { FDR adjusted } \\
p\end{array}$ & $\begin{array}{c}\text { Mapped } \\
\text { genes }\end{array}$ & $\begin{array}{c}\text { Fold } \\
\text { enrichment }\end{array}$ & $\begin{array}{c}\text { FDR adjusted } \\
p\end{array}$ \\
\hline $\begin{array}{l}\text { Cytokine-cytokine receptor } \\
\text { interaction }\end{array}$ & 25 & 3.0 & $4.3 E-04$ & 38 & 2.5 & $3.0 E-05$ \\
\hline Hematopoietic cell lineage & 13 & 4.4 & $3.8 E-03$ & 15 & 2.7 & $2.1 E-02$ \\
\hline Lysosome & 14 & 3.2 & $2.1 E-02$ & 34 & 4.2 & $2.9 E-10$ \\
\hline Rheumatoid arthritis & 12 & 3.7 & $2.3 E-02$ & 21 & 3.5 & $7.0 E-05$ \\
\hline Malaria & 9 & 4.6 & $2.6 E-02$ & 14 & 3.9 & $1.4 E-03$ \\
\hline Phagosome & 15 & 2.7 & $3.9 E-02$ & 26 & 2.5 & $1.1 E-03$ \\
\hline Pertussis & 10 & 3.7 & $4.4 E-02$ & 20 & 3.9 & $4.0 E-05$ \\
\hline NF-kappa B signaling pathway & & & & 19 & 3.1 & $1.3 E-03$ \\
\hline $\begin{array}{l}\text { Transcriptional misregulation in } \\
\text { cancer }\end{array}$ & & & & 26 & 2.4 & $1.6 E-03$ \\
\hline $\begin{array}{l}\text { Chagas disease (American } \\
\text { trypanosomiasis) }\end{array}$ & & & & 20 & 2.7 & $3.3 E-03$ \\
\hline Leishmaniasis & & & & 14 & 3.3 & $6.6 E-03$ \\
\hline TNF signaling pathway & & & & 19 & 2.5 & $8.5 E-03$ \\
\hline Salmonella infection & & & & 16 & 2.8 & $8.8 E-03$ \\
\hline $\begin{array}{l}\text { Complement and coagulation } \\
\text { cascades }\end{array}$ & & & & 14 & 2.8 & $2.1 E-02$ \\
\hline Mineral absorption & & & & 10 & 3.4 & $3.1 E-02$ \\
\hline Osteoclast differentiation & & & & 20 & 2.2 & $3.2 E-02$ \\
\hline Legionellosis & & & & 12 & 2.8 & $4.4 E-02$ \\
\hline Pentose phosphate pathway & & & & 7 & 4.6 & $4.6 E-02$ \\
\hline Histidine metabolism & & & & 7 & 4.6 & $4.6 E-02$ \\
\hline
\end{tabular}

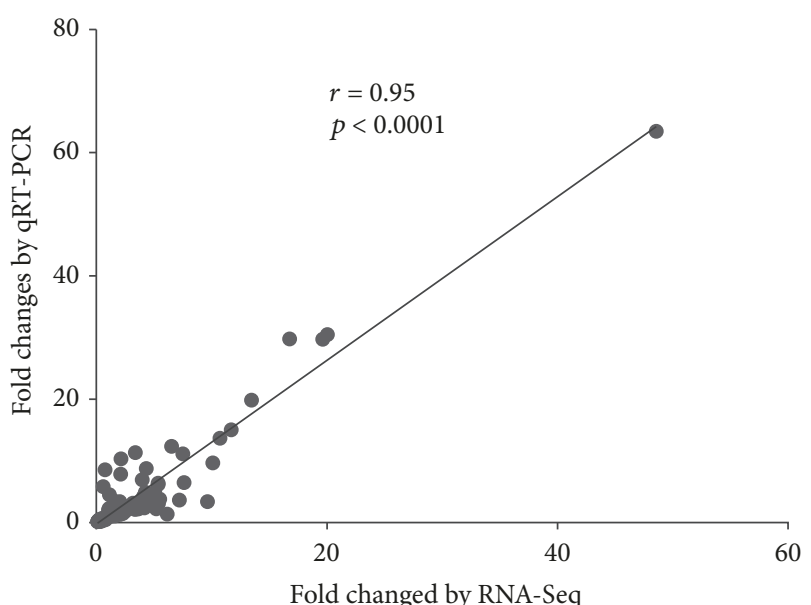

FIGURE 3: Correlation of gene fold changes between RNAsequencing (RNA-Seq) and qRT-PCR analysis.

route to cell death in a model of drug-induced ALF and therefore merits further study. In addition to pyroptosis, necrapoptosis, also known as aponecrosis or apoptotic necrosis, is an important proinflammatory cell death pattern that shared common features and pathways with both apoptosis and necrosis $[33,34]$. Also, this cell death pattern was found in liver injury $[35,36]$. The necrapoptosis GO term or KEGG pathway was not enriched or mapped in this study. The possible reason might be that this cell demise pattern has not been annotated in databases of GO (http://geneontology.org/) and KEGG (http://www.kegg.jp/), for we cannot retrieve it in either of two databases so far. However, GO term, the adenosine triphosphate (ATP) hydrolysis coupled proton transport, was enriched in this study. ATP has been proved as a key factor to determine the way out of necrapoptosis [34]. This might verify from another aspect in transcriptome level that necrapoptosis is an important cell death pattern involved in the progression of ALF.

Autophagy is a lysosomal pathway tasked with the process of self-degradation of cellular components by the sequestration of these components in double-membrane autophagosomes [37]. It has been widely reported that autophagy plays an important role in cancer and other chronic diseases of the organs [38-42]. Autophagy is an important current research topic in models of liver disease [43-45]. However, currently there are limited data on the role of autophagy in the progression of ALF $[46,47]$. In this study, GO terms such as autophagy, regulation of autophagy, and phagocytic-vehicle were all enriched. Two KEGG pathways of lysosomal and phagosome regulation were mapped. These results provide another potential avenue of transcriptome-level research on the influence of autophagy on the progression of ALF. 
The role of fibrosis in the progression of chronic liver disease has been widely studied. Although fibrosis is observed in ALF [48], an understanding of the underlying mechanisms remains limited. GO terms related to the collagen-related component of liver fibrosis, such as collagen catabolic processes and collagen binding, were also enriched in this study. Semaphorin families are regulators of the progression of fibrosis in chronic liver diseases $[49,50]$. However, the role of semaphorin families in ALF remains unknown. Semaphorin receptor activity GO terms were also found in this study, which constitutes another interesting avenue for research.

In conclusion, this study identified dramatic changes in the PBMC transcriptome predominantly related to immune responses in ALF. Enriched GO terms related to coagulation dysfunction, hepatic encephalopathy, and mapped KEGG pathways of diseases characterised by liver injury demonstrated that the PBMC transcriptome reflects the features of ALF. The enrichment of GO terms related to cell death and fibrosis indicates that PBMC transcriptome analysis is a useful method to elucidate potential key gene sets involved in ALF progression. Thus, a better understanding of the gene sets identified in this study may contribute to ALF prevention or treatment.

\section{Conflicts of Interest}

The authors declare no conflicts of interest.

\section{Authors' Contributions}

YiMin Zhang and Li Shao contributed equally to this paper; LanJuan Li and YiMin Zhang designed the study; YiMin Zhang, Ning Zhou, JianZhou Li, Yu Chen, and Juan Lu performed experiments; Li Shao, ErMei Chen, Jie Wang, and ZhongYang Xie collected the data. YiMin Zhang and Li Shao analysed the data and wrote the paper.

\section{Acknowledgments}

This work was supported by China National Science and Technology Major Project, no. 2017ZX10202202; National Natural Science Foundation of China, no. 81600497; and Zhejiang CTM Science and Technology Project, no. 2011ZB061.

\section{Supplementary Materials}

Supplementary Table 1: list of differentially expressed genes identified in failure group; Supplementary Table 2: list of differentially expressed genes identified in dying group; Supplementary Table 3: product sizes and the primers used for qRT-PCR; Supplementary Table 4: gene fold change tested by qRT-PCR and RNA-Seq. (Supplementary Materials)

\section{References}

[1] J. Polson and W. M. Lee, "AASLD position paper: The management of acute liver failure," Hepatology, vol. 41, no. 5, pp. 11791197, 2005.
[2] S. L. Flamm, Y.-X. Yang, S. Singh et al., "American Gastroenterological Association Institute Guidelines for the Diagnosis and Management of Acute Liver Failure," Gastroenterology, vol. 152, no. 3, pp. 644-647, 2017.

[3] J. Wendon, J. Cordoba, A. Dhawan et al., "EASL clinical practical guidelines on the management of acute (fulminant) liver failure," Journal of Hepatology, vol. 66, no. 5, pp. 1047-1081, 2017.

[4] T. A. Skvortsov, D. V. Ignatov, K. B. Majorov, A. S. Apt, and T. L. Azhikina, "Mycobacterium tuberculosis transcriptome profiling in mice with genetically different susceptibility to tuberculosis," Acta Naturae, vol. 5, no. 17, pp. 62-69, 2013.

[5] H. Kalam, M. F. Fontana, and D. Kumar, "Alternate splicing of transcripts shape macrophage response to Mycobacterium tuberculosis infection," PLoS Pathogens, vol. 13, no. 3, Article ID e1006236, 2017.

[6] C. Rippe, B. Zhu, K. K. Krawczyk et al., "Hypertension reduces soluble guanylyl cyclase expression in the mouse aorta via the Notch signaling pathway," Scientific Reports, vol. 7, no. 1, article no. 1334, 2017.

[7] J. Chesne, R. Danger, K. Botturi et al., "Systematic analysis of blood cell transcriptome in EndStage chronic respiratory diseases," PLoS ONE, vol. 9, no. 10, Article ID e109291, 2014.

[8] S. Tattermusch, J. A. Skinner, D. Chaussabel et al., "Systems biology approaches reveal a specific interferon-inducible signature in HTLV-1 associated myelopathy," PLoS Pathogens, vol. 8, no. 1, Article ID e1002480, 2012.

[9] T. D. Querec, R. S. Akondy, E. K. Lee et al., "Systems biology approach predicts immunogenicity of the yellow fever vaccine in humans," Nature Immunology, vol. 10, no. 1, pp. 116-125, 2009.

[10] J. S. Tsang, P. L. Schwartzberg, Y. Kotliarov et al., "Global analyses of human immune variation reveal baseline predictors of postvaccination responses," Cell, vol. 157, no. 2, pp. 499-513, 2014.

[11] N. Zhou, J. Li, Y. Zhang et al., "Efficacy of coupled low-volume plasma exchange with plasma filtration adsorption in treating pigs with acute liver failure: a randomised study, Journal of Hepatology, vol. 63, no. 2, pp. 378-387, 2015.

[12] C. Trapnell, A. Roberts, L. Goff et al., "Differential gene and transcript expression analysis of RNA-seq experiments with TopHat and Cufflinks," Nature Protocols, vol. 7, no. 3, pp. 562$578,2012$.

[13] D. W. Huang, B. T. Sherman, and R. A. Lempicki, "Systematic and integrative analysis of large gene lists using DAVID bioinformatics resources," Nature Protocols, vol. 4, no. 1, pp. 44-57, 2009.

[14] R. Vemuganti, V. R. Silva, S. L. Mehta, and A. S. Hazell, "Acute liver failure-induced hepatic encephalopathy is associated with changes in microRNA expression profiles in cerebral cortex of the rat," Metabolic Brain Disease, vol. 29, no. 4, pp. 891-899, 2014.

[15] R. B. Meeker, D. C. Bragg, W. Poulton, and L. Hudson, “Transmigration of macrophages across the choroid plexus epithelium in response to the feline immunodeficiency virus," Cell and Tissue Research, vol. 347, no. 2, pp. 443-455, 2012.

[16] Y. Huang, X.-Y. Zhu, M.-R. Du, and D.-J. Li, "Human trophoblasts recruited $\mathrm{T}$ lymphocytes and monocytes into decidua by secretion of chemokine CXCL16 and interaction with CXCR6 in the first-trimester pregnancy," The Journal of Immunology, vol. 180, no. 4, pp. 2367-2375, 2008.

[17] T. Zhang, L. Tian, G. Hu, K. Teng, and X. Mu, "Microvascular endothelial cells play potential immunoregulatory roles in the immune response to foot-and-mouth disease vaccines," Cell Biochemistry \& Function, vol. 29, no. 5, pp. 394-399, 2011. 
[18] F. Chen, H.-H. Zhu, L.-F. Zhou et al., "Genes related to the very early stage of ConA-induced fulminant hepatitis: A gene-chipbased study in a mouse model," BMC Genomics, vol. 11, no. 1, article no. 240, 2010.

[19] N. J. Taylor, A. Nishtala, G. K. Manakkat Vijay et al., "Circulating neutrophil dysfunction in acute liver failure," Hepatology, vol. 57, no. 3, pp. 1142-1152, 2013.

[20] P. E. Marques, S. S. Amaral, D. A. Pires et al., "Chemokines and mitochondrial products activate neutrophils to amplify organ injury during mouse acute liver failure," Hepatology, vol. 56, no. 5, pp. 1971-1982, 2012.

[21] S. Zhang, N. Yang, S. Ni et al., "Pretreatment of lipopolysaccharide (LPS) ameliorates D-GalN/LPS induced acute liver failure through TLR4 signaling pathway," International Journal of Clinical and Experimental Pathology, vol. 7, no. 10, pp. 66266634, 2014.

[22] N. Shah, M. Montes De Oca, M. Jover-Cobos et al., "Role of toll-like receptor 4 in mediating multiorgan dysfunction in mice with acetaminophen induced acute liver failure," Liver Transplantation, vol. 19, no. 7, pp. 751-761, 2013.

[23] R. Sehgal, S. Patra, P. David et al., "Impaired monocytemacrophage functions and defective toll-like receptor signaling in hepatitis E virus-infected pregnant women with acute liver failure," Hepatology, vol. 62, no. 6, pp. 1683-1696, 2015.

[24] L. Collin, P. Moulin, M. Jungers, and A. P. Geubel, "Epstein-Barr virus (EBV)-induced liver failure in the absence of extensive liver-cell necrosis: A case for cytokine-induced liver dysfunction?” Journal of Hepatology, vol. 41, no. 1, pp. 174-175, 2004.

[25] J. Li, X. Zhu, F. Liu et al., "Cytokine and autoantibody patterns in acute liver failure," Journal of Immunotoxicology, vol. 7, no. 3, pp. 157-164, 2010.

[26] Z. Cao, F. Li, X. Xiang et al., "Circulating cell death biomarker: Good candidates of prognostic indicator for patients with hepatitis B virus related acute-on-chronic liver failure," Scientific Reports, vol. 5, Article ID 14240, 2015.

[27] H. Bantel and K. Schulze-Osthoff, "Mechanisms of cell death in acute liver failure," Frontiers in Physiology, vol. 3, article no. 79, 2012.

[28] J. An, C. Harms, G. Lättig-Tünnemann et al., “TAT-apoptosis repressor with caspase recruitment domain protein transduction rescues mice from fulminant liver failure," Hepatology, vol. 56, no. 2, pp. 715-726, 2012.

[29] P. Zhang, M. Zhang, M. Wan et al., “Tamoxifen Attenuates Lipopolysaccharide/Galactosamine-induced acute liver failure by antagonizing hepatic inflammation and apoptosis," Immunological Investigations, vol. 46, no. 3, pp. 284-294, 2017.

[30] K. R. Bortoluci and R. Medzhitov, "Control of infection by pyroptosis and autophagy: Role of TLR and NLR," Cellular and Molecular Life Sciences, vol. 67, no. 10, pp. 1643-1651, 2010.

[31] Y. Geng, Q. Ma, Y.-N. Liu et al., "Heatstroke induces liver injury via IL-1 $\beta$ and HMGB1-induced pyroptosis," Journal of Hepatology, vol. 63, no. 3, article no. 5648, pp. 622-633, 2015.

[32] Y. L. Chen, G. Xu, X. Liang et al., "Inhibition of hepatic cells pyroptosis attenuates CLP-induced acute liver injury," American journal of translational research, vol. 8, no. 12, pp. 5685-95, 2016.

[33] L. Formigli, L. Papucci, A. Tani et al., "Aponecrosis: morphological and biochemical exploration of a syncretic process of cell death sharing apoptosis and necrosis," Journal of Cellular Physiology, vol. 182, no. 1, pp. 41-49, 2000.

[34] J. J. Lemasters, T. Qian, L. He et al., "Role of mitochondrial inner membrane permeabilization in necrotic cell death, apoptosis, and autophagy," Antioxidants \& Redox Signaling, vol. 4, no. 5, pp. 769-781, 2002.

[35] A. M. El-Gibaly, C. Scheuer, M. D. Menger, and B. Vollmar, "Improvement of rat liver graft quality by pifithrin- $\alpha$-mediated inhibition of hepatocyte necrapoptosis," Hepatology, vol. 39, no. 6, pp. 1553-1562, 2004.

[36] H. Jaeschke and J. J. Lemasters, "Apoptosis versus oncotic necrosis in hepatic ischemia/reperfusion injury," Gastroenterology, vol. 125, no. 4, pp. 1246-1257, 2003.

[37] C. He and D. J. Klionsky, "Regulation mechanisms and signaling pathways of autophagy," Annual Review of Genetics, vol. 43, pp. 67-93, 2009.

[38] Z. Zhong, E. Sanchez-Lopez, and M. Karin, "Autophagy, inflammation, and immunity: a troika governing cancer and its treatment," Cell, vol. 166, no. 2, pp. 288-298, 2016.

[39] J. Moscat and M. T. Diaz-Meco, "p62 at the crossroads of autophagy, apoptosis, and cancer," Cell, vol. 137, no. 6, pp. 10011004, 2009.

[40] Z. V. Wang, B. A. Rothermel, and J. A. Hill, "Autophagy in hypertensive heart disease," The Journal of Biological Chemistry, vol. 285, no. 12, pp. 8509-8514, 2010.

[41] T. Kimura, Y. Isaka, and T. Yoshimori, "Autophagy and kidney inflammation," Autophagy, vol. 13, no. 6, pp. 997-1003, 2017.

[42] K. Mizumura, S. Cloonan, and M. E. Choi, "Autophagy: Friend or Foe in Lung Disease?" Annals of the American Thoracic Society, vol. 13, 1, pp. S40-S47, 2016.

[43] X.-M. Yin, W.-X. Ding, and W. Gao, "Autophagy in the liver," Hepatology, vol. 47, no. 5, pp. 1773-1785, 2008.

[44] C. Zhang and A. M. Cuervo, "Restoration of chaperone-mediated autophagy in aging liver improves cellular maintenance and hepatic function," Nature Medicine, vol. 14, no. 9, pp. 959-965, 2008.

[45] P.-E. Rautou, A. Mansouri, D. Lebrec, F. Durand, D. Valla, and R. Moreau, "Autophagy in liver diseases," Journal of Hepatology, vol. 53, no. 6, pp. 1123-1134, 2010.

[46] F. Ren, L. Zhang, X. Zhang et al., "Inhibition of glycogen synthase kinase 3 beta promotes autophagy to protect mice from acute liver failure mediated by peroxisome proliferatoractivated receptor alpha," Cell death \& disease, vol. 7, article e2151, 2016.

[47] L. Jin, H. Gao, J. Wang et al., "Role and regulation of autophagy and apoptosis by nitric oxide in hepatic stellate cells during acute liver failure," Liver International, vol. 37, no. 11, pp. 1651$1659,2017$.

[48] Y. He, L. Jin, J. Wang, Z. Yan, T. Chen, and Y. Zhao, "Mechanisms of fibrosis in acute liver failure," Liver International, vol. 35, no. 7, pp. 1877-1885, 2015.

[49] T. Yagai, A. Miyajima, and M. Tanaka, "Semaphorin 3E secreted by damaged hepatocytes regulates the sinusoidal regeneration and liver fibrosis during liver regeneration," The American Journal of Pathology, vol. 184, no. 8, pp. 2250-2259, 2014.

[50] S. De Minicis, C. Rychlicki, L. Agostinelli et al., "Semaphorin 7A contributes to TGF- $\beta$-mediated liver fibrogenesis," The American Journal of Pathology, vol. 183, no. 3, pp. 820-830, 2013. 


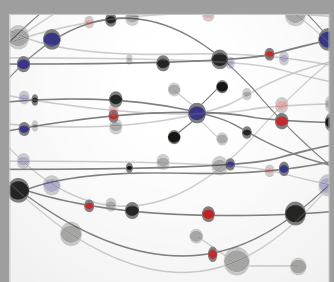

The Scientific World Journal
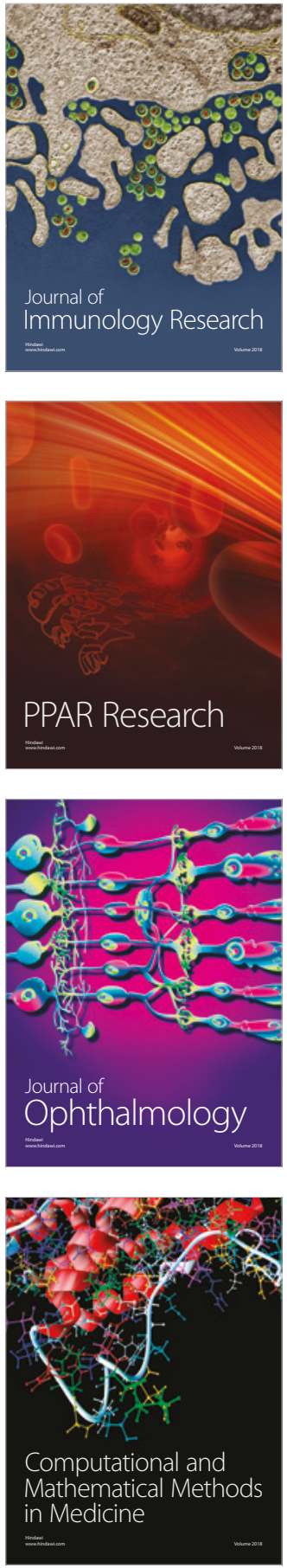

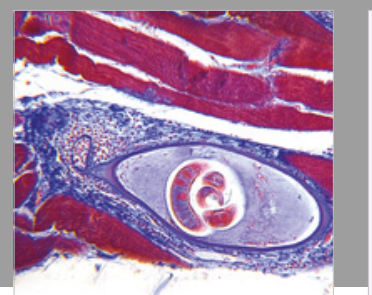

Gastroenterology Research and Practice

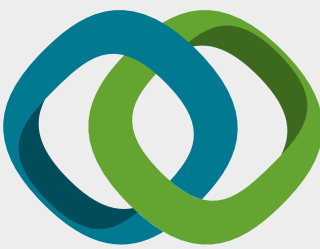

\section{Hindawi}

Submit your manuscripts at

www.hindawi.com
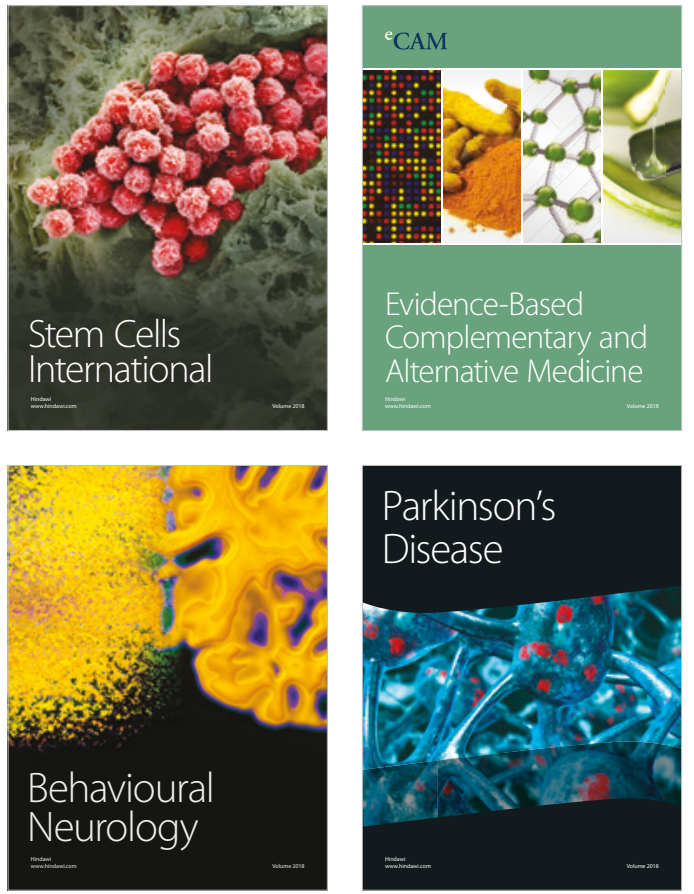

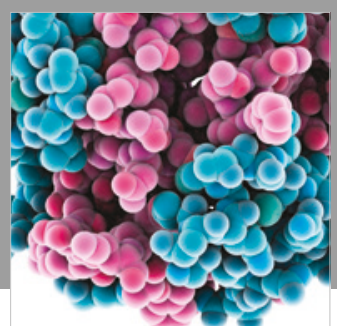

ournal of

Diabetes Research

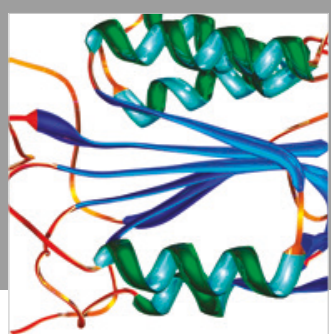

Disease Markers
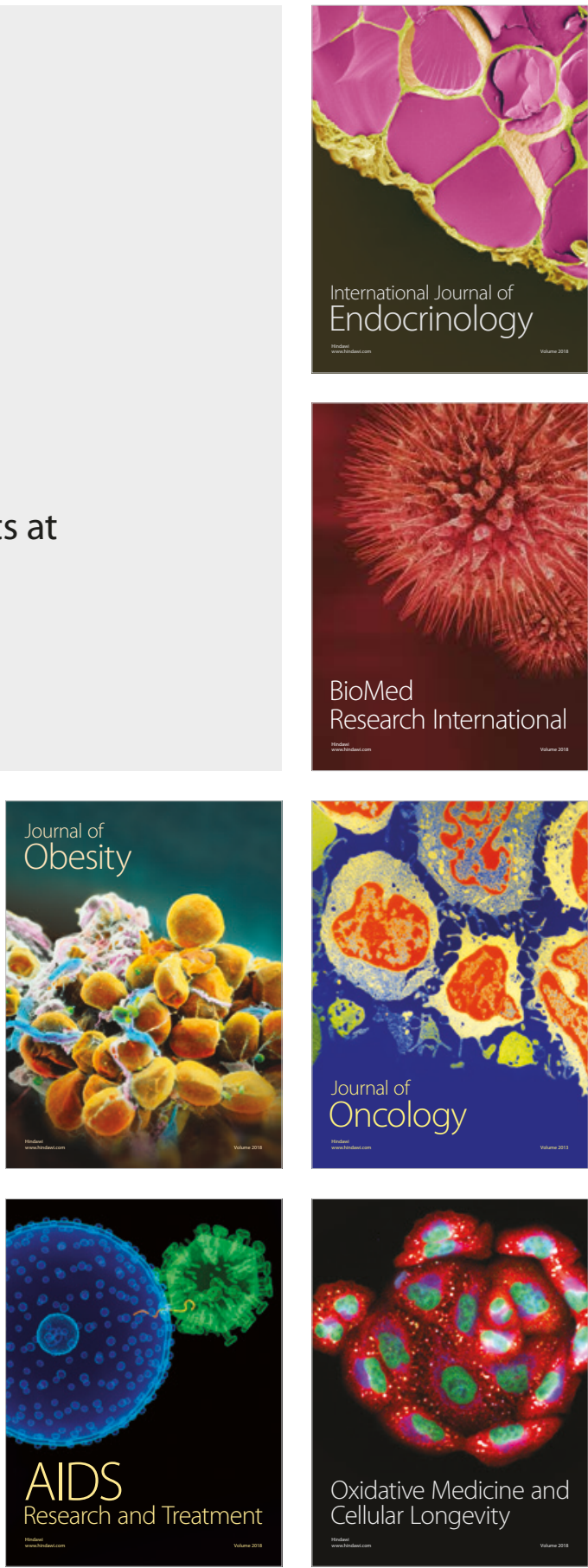